\title{
An Analytical Study on Aberrant Ventricular Conduction
}

\section{Part I. A General Rule}

\author{
Satoru MURAO, M. D., Akio KUROIWA, M.D., \\ Tsuneaki SUGIMOTO, M. D., Kenichi, HARUMI, M. D., \\ and Kazuzo KATO, M. D.
}

In order to clarify the mechanism of aberrant ventricular conduction, the quantitative relationship between the ventricular cycle of a supraventricular impulse and the preceding ventricular cycle was analyzed graphically on 15 cases with supraventricular arrhythmia. A fairly sharp boundary curve, resembling the standard QT curve, between the conditions causing aberrantly and normally conducted beats, was obtained in each case. This result shows that the main factor of aberrant ventricular conduction is the refractory period of the intraventricular conduction system.

$I^{\mathrm{T}}$

$T$ is well known that the aberrant ventricular conduction frequently occurs in the various types of supraventricular arrhythmia, when a short ventricular cycle follows a relatively long one. Since this phenomenon was first reported by Lewis ${ }^{1)}$ as aberrant ventricular response, many reports have been presented concerning its clinical significance and Inechanism.

Though Scherf, ${ }^{2)}$ Gouaux and Ashman, ${ }^{3}$ and Langendorf and Pick (5) showed as its mechanism that the refractory period following a long ventricular cycle is too long for the following supraventricular impulse with a short cycle to conduct the ventricle normally. Since no study on the quantitative correlation between the ventricular cycle of supraventricular impulse and the preceding cycle has been reported on clinical materials, the present study was undertaken to clarify the problems.

\section{Matertals and Method}

Twelve cases of supraventricular premature beats, 2 cases of atrial fibrillation and one case of interference dissociation between sinus bradyarrhythmia

From the Second Department of Internal Medicine, Faculty of Medicine, University of Tokyo, Tokyo. 
and nodal escaped rhythm, whose electrocardiograms showed many aberrantly and normally conducted beats, were analyzed. In 2 cases of atrial fibrillation, digilalis was not given and only one type of bizarre ventricular complex, with complete and/or incomplete right bundle branch block pattern was shown. All these ventricular complexes could be differentiated from ventricular premature beats by the criteria by Langendorf and Pick. ${ }^{5)}$

Every ventricular complex was plotted as one point in a diagram, the ordinate of which represents a ventricular cycle of the supraventricular impulse following a long cycle, and the abscissa of which represents the preceding long cycle. By this diagram, it can be examined whether a boundary condition of aberrant ventricular conduction may be found or not in cach ventricular cycle.

\section{Results}

Case 1. 65-year-old man with an old myocardial infarction. The EGG (Fig. 1a) shows multiple supraventricular premature beats with varied

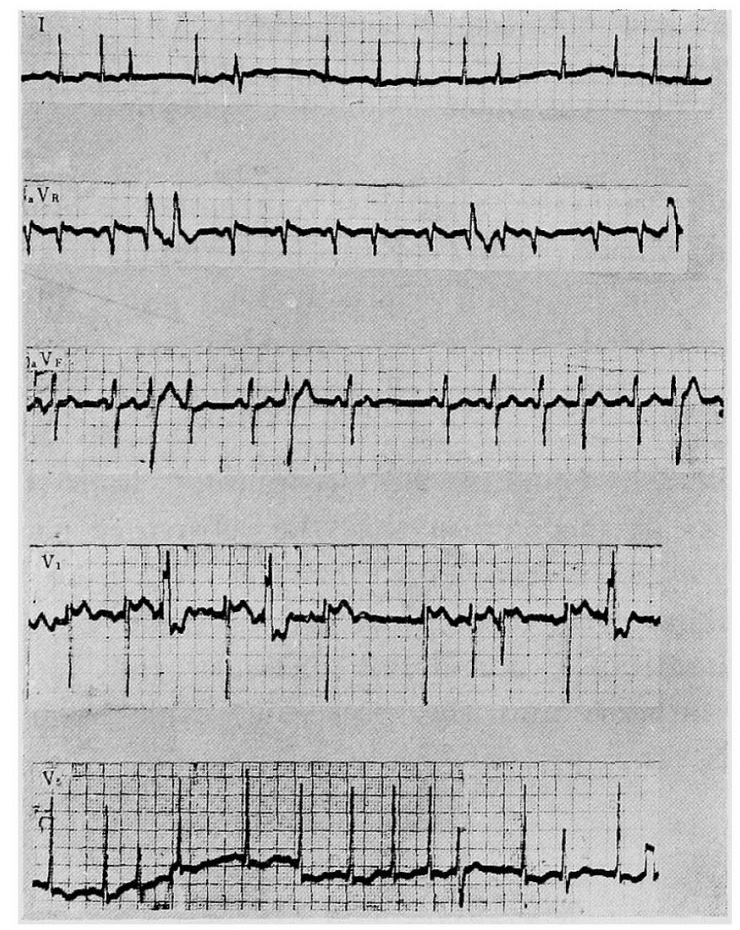

Fig. 1a.

coupling and preceding interval, and many aberrantly and normally conducted beats. Fig. $1 \mathrm{~b}$ is the analytical diagram of this case, where the signals of small circle, $\mathrm{x}$ and dot represent normally, less aberrantly and aberrantly conducted beats, respectively. 
In this figure, the area of the aberrant ventricular conduction is under that of normal conduction as a whole, and a boundary line showing upper limit of the condition causing aberrant conduction can be drawn fairly sharply, with some exceptions. This curve is alike an exponential curve, such as the standard QT curve referring to each ventricular cycle.

Case 2. 48-year-old man with hypertension and cerebral hemorrhage. The EGG (Fig. 2a) shows multiple atrial premature beats, and 2 types

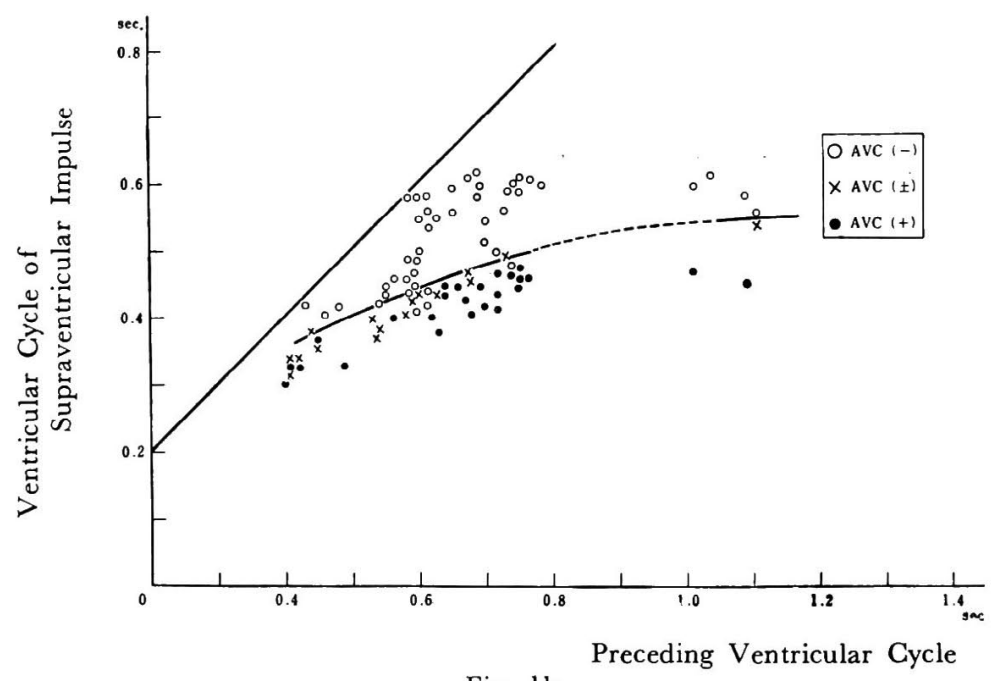

Fig. 1b.

of aberrantly conducted ventricular complex, that is, both right and left bundle branch block pattern. As seen in Fig. 2b, the boundary line is fairly sharp when the ventricular preceding cycle is long, but it is not the case when the preceding ventricular cycle is short especially when these beats appeared in succession.

Case 3. 35-year-old man with mitral valvular disease. The ECG (Fig. 3a) shows atrial fibrillation, aberrantly conducted beats with com-

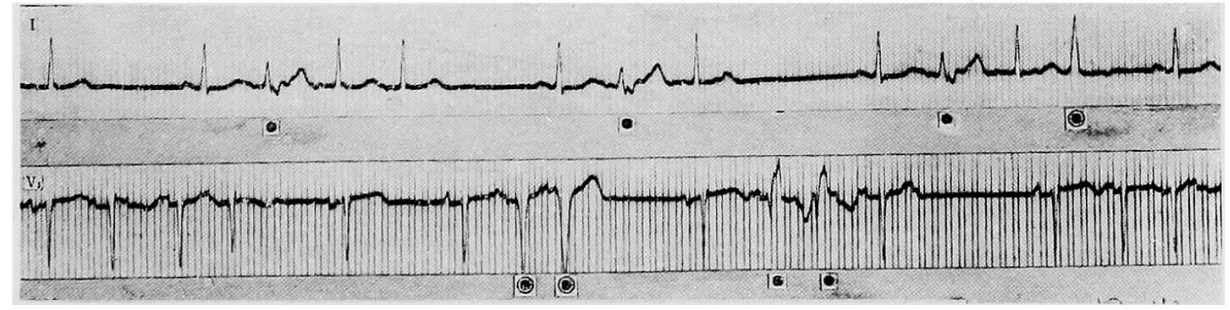

Fig. 2a.

plete and incomplete right bundle branch block pattern, and short runs of the bizarre ventricular complexes. Fig. $3 \mathrm{~b}$ represents the analysis of 


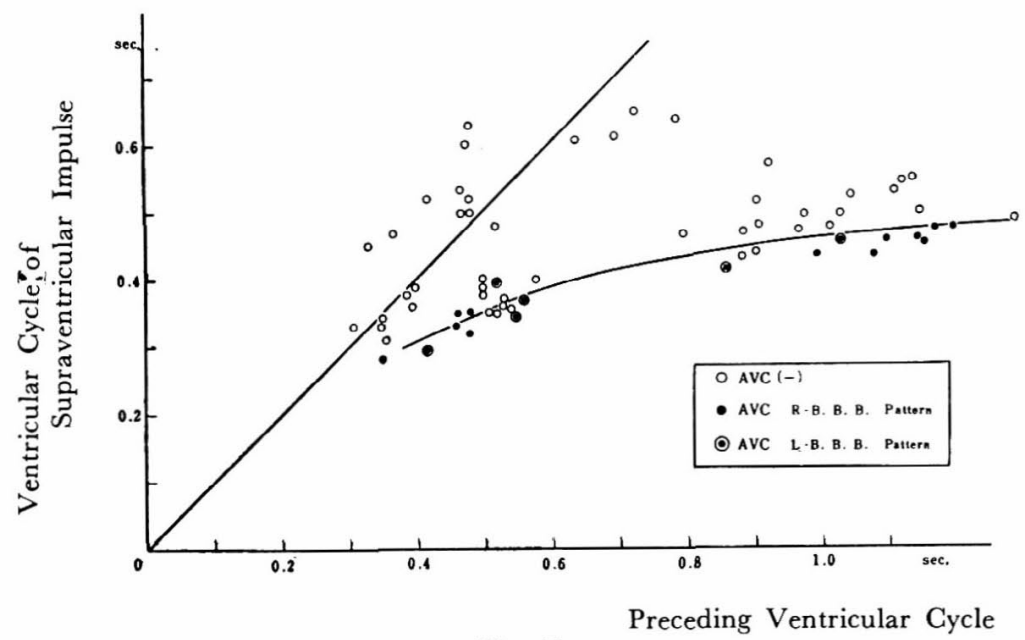

Fig. 2b.

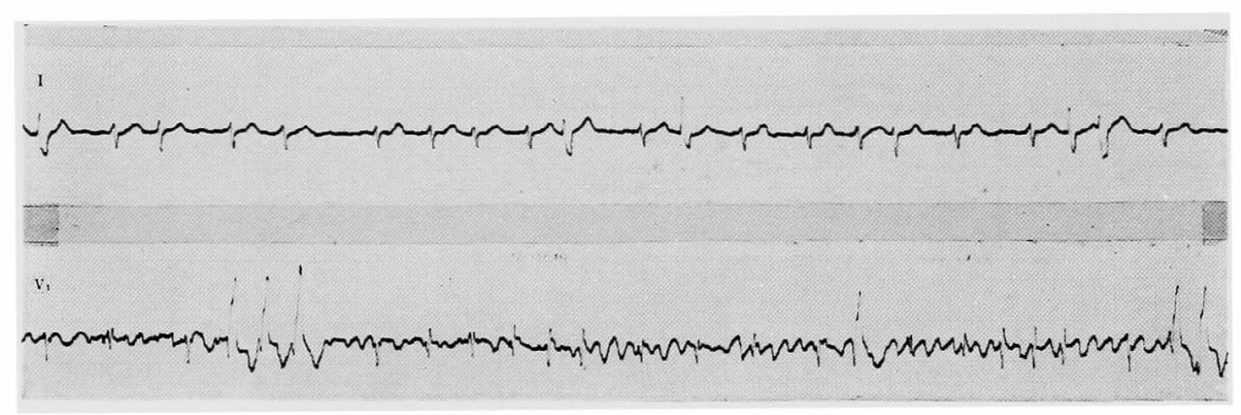

Fig. 3a.

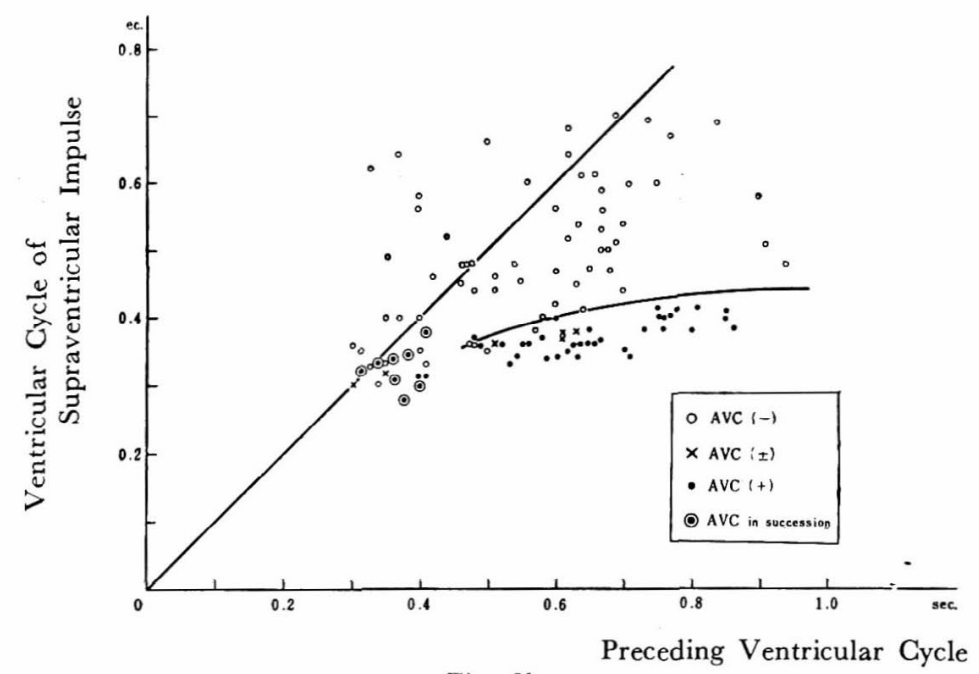

Fig. 3b. 
the boundary condition, and shows almost the same findings as in case 2 .

The analytical studies on other cases are almost the same, and a boundary line can be traced in a wide range of ventricular cycle in each case, if some irregularities are ignored. Fig. 4 represents the summary of

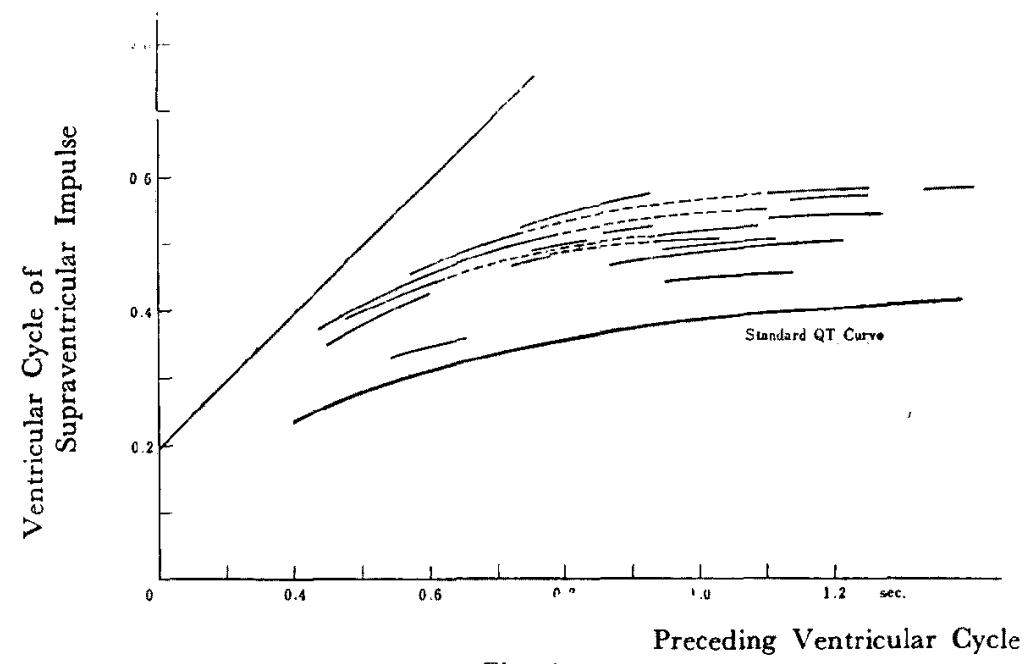

Fig. 4.

the analytical studies in all cases. As seen in this figure, the course of the boundary curves are essentially similar, though the level of this curve is somewhat different case by case. Furthermore, these curves occupy comparatively upper position above the standard QT curve in this analytical figure.

\section{Discussion}

Though the phenomenon of aberrant ventricular conduction is well known since about 40 years ago, its precise mechanism has not been sufficiently clarified. In 1925, Lewis and Master ${ }^{6}$ demonstrated that the refractory period of atrioventricular conduction system was determined almost only by the length of the immediately preceding cycle, and Mendez and associates? ${ }^{\text {? }}$ clarified the similar relationship on the ventricular refractory period. According to these experimental studies, more than one preceding ventricular cycles, regular or irregular, have almost no cumulative effect on the refractory period to be questioned.

This is the theoretical background on which the present study was performed.

Fig. 4 shows clearly that the condition in which aberrant conduction occurs, is mostly determined by the relation between the preceding cycle and the following cycle and that the boundary line is represented as a 
limited curve resembling the standard QT curve. This may be the clinical representation concerning with the refractory period of ventricular conduction system, and may be understood analogously to the experimental studies concerning the refractory period of atrioventricular conduction system by Lewis and Master and that of ventricular muscle by Mendez and associates.

A few factors, which may modify the boundary condition in this diagram must be considered here. The first is the fact that the ventricular cycle of the premature supraventricular impulse can not always represent the true time interval between the 2 ventricular excitations, but it may contain the silent intraventricular conduction time. This phenomenon was demonstrated in the cases represented by Burchell ${ }^{8}$ and Holzmann, ${ }^{9)}$ whose ECGs show supraventricular arrhythmias with 2 types of aberrantly conducted beats, left and right bundle branch block pattern. This silent intraventricular conduction time must be considered to disturb the sharp delineation of boundary conditions in some cases. This factor will be discussed in the following paper.

The second factor is concerning with the irregularity of boundary conditions frequently seen in the short preceding cycle especially in the succession of aberrantly conducted beats. This phenomenon is well explained by Gouaux and Ashman at least in some parts. On this point, there remains some uncertain problems that will be discussed in the following paper.

The third problem is the difference of the level of the boundary line in each case. The presence or severity of some pathological process in the ventricular conduction system ${ }^{10), 11}$ may be one factor for this problem, but the individual difference of refractoriness may play a role. The number of cases in this study is too small to analyse these factors. It is worth to be mentioned that the level of boundary lines is distinctly high above the standard QT curve in all cases. This shows that the refractory period of the ventricular conduction system is longer than that of the ventricular muscle.

Though these problems remain unsettled, a general rule of the quantitative relationship between preceding ventricular cycle and following cycle in the phenomenon of aberrant ventricular conduction is fairly clearly demonstrated in this clinical study, and this analytical method may contribute to the differentiation of ventricular premature beat from aberrant ventricular conduction in some cases of atrial fibrillation.

\section{Summary}

(1) In 15 cases of supraventricular arrhythmia with many aber- 
rantly conducted beats, a relationship between the ventricular cycle of a supraventricular impulse and the preceding ventricular cycle was analysed, and the fairly limited boundary line concerning aberrant ventricular conduction was able to be traced in an analytical diagram in every case.

(2) Though the level of this boundary curve was somewhat varied in each case, its position in the analytical diagram was higher than the standard QT curve, and its figure was similar to the standard QT curve as a whole in every case.

(3) These results clearly showed that the main factor for aberrant ventricular conduction is the length of refractory period of the intraventricular conduction system following the preceding ventricular cycle.

(4) Several factors which might modify such a relationship were briefly discussed.

\section{REFERENCES}

1. Lewis, T.: Mechanism and Graphic Registration of the Heart Beat, Shaw, London, 1925.

2. Scherf, D.: Wien. Arch. Inn. Med. 18: 403, 1929.

3. Gouaux, J. L. and Ashman, R.: Am. Heart J. 34: 366, 1947.

4. Langendorf, R.: Am. Heart J. 41: 700, 1951.

5. Langendorf, R. and Pick, A.: Prog. Cardiov. Dis. 2: 706, 1960.

6. Lewis, T. and Master, A. M.: Heart 12: 209, 1925.

7. Mendez, C., Gruhzit, C. G., and Moe, G. K.: Am. J. Physiol. 184: 287, 1956.

8. Burchell, H. B.: Brit. Heart J. 11 : 230, 1949.

9. Holzmann, M.: Die Rhythmusstörungen des Herzens, Handbuch der Inn. Med. 4te Auflage, IX/2, Springer-Verlag, Berlin · Göttingen • Heidelberg, p. 52, 1960.

10. Vessel, H.: Am. J. Med. Sc. 202 : 198, 1941.

11. Berliner, K. and Lewithin, L. P.: Am. Heart J. 29, 449, 1945. 